\title{
Flammulina Velutipes (Curt.: Fr.) Singer: An Edible Mushroom in Northern Forest of Iran and its Antagonistic Activity Against Selected Plant Pathogenic Fungi
}

\author{
Ali Borhani \\ Agriculture and Natural Research Center of Mazandaran \\ Passand forest and rangeland research station, P.O. Box 167 Behshar, Iran \\ Susanna M Badalyan \\ Laboratory of Fungal biology and biotechnology \\ Yerevan State University, Aleg Manoogian St. 375025, Yerevan, Armenia \\ Narine N Garibyan \\ Laboratory of Fungal biology and biotechnology \\ Yerevan State University, Aleg Manoogian St. 375025, Yerevan, Armenia \\ Saied Ali Mosazadeh \\ Agriculture and Natural Research Center of Mazandaran \\ Passand forest and rangeland research station, P.O. Box 167 Behshar, Iran \\ Esmaeil Yasari (Corresponding Author) \\ Asstt Prof, Payame Noor University, Sari, Iran \\ E-mail: e_yassari@yahoo.com
}

Received: September 02, 2010 Accepted: September 14, 2010 doi:10.5539/ijb.v3n2p162

\begin{abstract}
Flammulina velutipes is known as edible and medicinal mushroom. The fungus grow on living trees of Zelkova carpinifolia and dead trees of Diospyros lotus (Stand and fallen) in Behshar forest area. These plants were found as new hosts for F. velutipes. In vitro antagonistic activity of this mushroom against Bipolaris sorokiniana, Ophiostoma ulmi, Pestalotiopsis funerea, Fusarium culmurum, and Fusarium oxysporum was evaluated on MEA as dual culture. Colonies of the fungus were white farinaceous and powdery on MEA, later turns yellow. Daily growth of colony was measured $5.36 \mathrm{~mm}$ on MEA at $25^{\circ} \mathrm{C}$. Growth coefficient was indicated 36.64 and $90 \mathrm{~mm}$ Petriplate was covered up to 15 days after plating. The strongest and poorest antagonistic index have been seen against Ophiostoma ulmi and Bipolaris sorokiniana, respectively.
\end{abstract}

Keywords: Flammulina velutipes, Antagonistic activities, Bio characteristics, North of Iran, Edible mushroom

\section{Introduction}

Many mushrooms have been valued throughout the world as food and medicine. Europeans have always appreciated their gastronomic value. In Japan pushcart venders sell medicinal mushrooms on the street which are regularly used in diet to maintain health and promote longevity. However fear of mushroom poisoning also exists to a great extent many parts of this earth, despite presence of edible mushroom cultures throughout Asia, many parts of Europe and USA (Smith and Sullivan, 2004). 
Flammulina velutipes (Curt.:Fr.) Sing is the second mushroom after Auricularia auricula (Bull.: Fr.) Wett. which has been grown since 800 A. D. (Chang and Miles, 1987). This mushroom is mainly cultivated for gourmet and tonic purposes. Initially cultivation of fungus was on logs, but in recent years it has been cultivated on sawdust in Japan, Taiwan and china. Hardwood sawdust is preferred to conifers for mycelial growth and the initiation of fruiting body primordial, but mixtures of the two are very satisfactory. This mushroom is popular in Asian countries, especially China, Japan, and South Korea. Production of this edible mushroom has been doubled from $1990\left(143,000 \mathrm{MT}^{*}\right)$ to 1997 (284,700 MT). Production in China was increased by 26 fold from $15,000 \mathrm{MT}$ in 1997 to 389,324 in 2002. In low-temperature fructification of mushroom takes place and it continues all the year under controlled conditions. Although this mushroom can only produce fruiting bodies in low- temperature condition it can be massively produced years around under controlled low- temperature or by rotating crops in places where the temperature is suitable (Chang and Miles, 2004).

Flammulina to family of Tricholomataceae comprises around 12 species, 4 varieties, and 2 forms commonly cultivated for food and medicinal properties (Hughes et al., 1999; Redhead and Petersen, 1999). The fungus is also known as winter mushroom or velvet stems with worldwide distribution (Chang and Miles, 2004).

Different groups of bioactive compounds such as polysaccharides, protein- glucan complex, sterols, lectins, peroxidases, laccases, cellulases and proteases, with medicinal and pharmaceutical properties (immunomodulating, antitumor, anti-oxidant, thrombolytic, fibrinolotic, antibacterial, antifungal, antiviral, haploidic, mitogenic were isolated from $F$. velutipes (Leung, 1997). Enokipodins A, B, C, and D are a-cuparene-type sesquiterpenoids antimicrobial metabolites produced in the stationary stage of $F$. velutipes mycelia development in malt extract broth (Melo et al., 2009). Several medicinal properties of mycelial and fruiting body samples of $F$. velutipes have been established in in-vitro experiment such as immunomodulatory effect via induction of cytokines and antifungal, antibacterial, antiviral, antioxidant, antiprotozoal, mitogenic activities (Badalyan and Hambardzumyan, 2001). A significant antifungal/antagonistic activity of several strains of $F$. velutipes against filamentous pathogenic fungi for men and/or animal has been reported (Badalyan, 2004). Polysaccharides and a low-weight protein-bound polysaccharide with high antitumor activity were also isolated from this mushroom (Ikekawa, 2001). Flammulin, a basic simple protein from $F$. velutipes is able to markedly inhibit tumor cells (Komatsu et al., 1963). An epidemiological study in Nagano Prefecture, Japan showed that cancer death rate among farmers producing $F$. velutipes was remarkably lower than that of other people in the Prefecture and other places in Japan (Ikekawa, 2001). Some drug or dietary supplement products have been made commercially for aniflammatory, antitumor and immunomodulating purpose (Wasser and Wies, 1999). Antagonistic activities of $F$. velutipes is reported against cereal pathogenic fungi such as Gaeumonnomyces graminis var. tritici, Bipolaris sorokiniana, Fusarium culmurum, Rhizoctonia cerealis (Badalyan et al., 2002).

The Fagus orientalis, Acer sp., Salix sp., Populus sp., Cydonia sp., Platanus orientalis and Malus sp have been reported as hosts of this mushroom in different parts of Iran (Saber 1998; Zokaie, 2001).

This report is a part of the results of project of investigation on biological characteristic and antifungal activities of several medicinal mushrooms from north of Iran. The aims of study were to identify their biological characteristics and antagonistic activities against plant pathogenic fungi in northern part of Iran.

\section{Materials and methods}

\subsection{Description of the research area}

Northern Iran comprised of three provinces Gillan (west), Mazandaran (center), Gollestan (east) and Hyrcanian forest zone which has encompassed humid commercial and industrial forests (Figure 1). This vegetation zone is a green belt stretching over the northern slops of Alborz mountain ranges and covers the southern coast of Caspian Sea. Alborz mountain interception between the Caspian Sea and Iran plateau has resulted in a climate with distinct vegetation cover. Hyrcanian forest stretch out from sea level up to an altitude of 3000 meters and encompasses different forest types and 80 woody species (trees and shrubs). (Sagheb-Talebi et al., 2005). The area is reach in hardwood, however there are only four genera of endemic softwood trees. Behshar, is a town in east of Mazandaran province, has been chosen in this study.

Forest trees species of this site comprise: Fagus orientalis .Acer velutinum, A. cappadocicum, Carpinus betulus, Quercus castaneaefolia, Diospyrus lotus, Zelkova carpinifolia, Almus glabra, Alnus glutinnosa, parrotia persica, Crataegus melanocarpa, Mespilus germanica. In low part and around the city there is forestry of specific kinds of conifer trees. Flora of the forest floor consists of: Rubus spp., Viola spp. Primula spp., Cyclamen coum, Mentha spp .and some other grass.

\subsection{Survey and Collection of samples}

A survey was carried out and samples were collected weekly during 2007-2009, in various forest areas at Behshar from an altitude of 30-1000 meters and some other forest areas of Mazandaran province occasionally. 
These samples were kept separately in each clean polyethylene bag with an label indicating the date of collection, place of collection, name of host, type of fruiting body and a brief description of the specimen.

\subsection{Purifications, culture conditions, investigation of morphological and Isolation}

Purifications, culture conditions, investigation of morphological and Isolation of $F$. velutipes was made using a tissue culture method on appropriate media Mate extract agar (MEA). In this order, a piece of cap (isolated from trunk of $Z$. carpinifolia) after surface disinfection with ethanol $96 \%$ was placed-onto media in Petri dish (90mm) with ventilation and to ovoid bacterial growth added $6 \mathrm{mg} / \mathrm{Lit} 25 \%$ lactic acid. Cultures were incubated in darkness at $25^{\circ} \mathrm{C}$. Growth characteristics of $F$. velutipes were studied by placing a $5 \mathrm{~mm}$ disc, cut from 3 day's old culture using a cork cutter, on the middle of $90 \mathrm{~mm}$ Petri dish with three replications. All measurements were obtained daily until the mycelia fully grow on the surface. The average growth rate (GR) and growth coefficient (GC) was calculated according to Buchalo's formule (1988): $G R=\Delta d / \Delta t$ and $G C=d g h / t$, where $d, g$, and $h$ are the diameter, density and height of the colony, respectively and $t$ is time.

Morphology and texture of colony were described by Stalper's scales and Nobel's codes (Nobles, 1965; Stalper, 1978). For micro morphological studies of vegetative mycelium, mycelial preparations of $F$. velutipes were obtained using slide culture method cultivated at $25^{\circ} \mathrm{C}$ on MEA (Badalyan and Sakeyan, 2004). The light microscope (Olympus $\mathrm{BH} 2$ ) equipped with calibrated drawing tube was used for this purpose.

To facilitate the formation of teleomorph $25 \mathrm{~mm} \times 200 \mathrm{~mm}$ tubes containing MEA were used. The tubes were inoculated with $F$. velutipes and incubated in dark at $25^{\circ} \mathrm{C}$ for ten days, then kept in a refrigerator at $6-8^{\circ} \mathrm{C}$ for 30 days. In addition, 7 pieces $(2-3 \times 7 \mathrm{~cm})$ of dried branches of Diospyros lotus were soaked in $17 \mathrm{~cm} \times 9 \mathrm{~cm}$ bottle for overnight to absorb enough moisture. Then sterilized for $30 \mathrm{~min}$ at $120^{\circ} \mathrm{C}$ and 1 atmosphere in autoclave, and were inoculated and incubated as in tube conditions.

\subsection{Antagonistic activities}

In order to evaluate antagonistic activities of $F$. velutipes five plant pathogenic fungi were used (table 1).

Competitive interactions between $F$. velutipes and the plant- pathogenic fungi were studied in dual cultures experiments by plotting on Malt Extract Agar (MEA Merck Germany, 48g/l) in Petri dishes. The plates were incubated at $25^{\circ} \mathrm{C}$ in darkness. Colony growth was examined daily under stereomicroscope to study interaction. A rating scale with 3 types $(A, B$ and $C)$ and 4 sub-type $\left(C_{A 1}, C_{B 1}, C_{A 2}\right.$ and $\left.C_{B 2}\right)$ for reactions was used to each fungus, where: $\mathrm{A}$, deadlock, mutual inhibition, in which the organism was able to overgrow the other after mycelial contact; $\mathrm{B}$, deadlock at a distance, means without mycelial contact; $\mathrm{C}$, replacement overgrow without initial deadlock; $\mathrm{C}_{\mathrm{A} 1}$ partial replacement after initial deadlock; $\mathrm{C}_{\mathrm{A} 2}$, complete replacement after initial deadlock; $\mathrm{C}_{\mathrm{B} 1}$, partial replacement after initial deadlock at distance; $\mathrm{C}_{\mathrm{B} 2}$, complete replacement after initial deadlock at distance. The following score was designed for each type or sub-type of reaction: $\mathrm{A}=1 ; \mathrm{B}=2 ; \mathrm{C}=3 ; \mathrm{C}_{\mathrm{A} 1}=3.5$; $\mathrm{C}_{\mathrm{B} 1}=4 ; \mathrm{C}_{\mathrm{A} 2}=4.5 ; \mathrm{C}_{\mathrm{B} 2}=5$. (Badalyan et al., 2002).

\section{Results}

\subsection{Ecology and morphology of fruiting body}

Fruiting body formation of $F$. velutipes varied during the years of study. The caps were observed from middle of October to middle of April on trunk of Diospyros lotus. Development of numbers of fruiting body varied on two hosts; D. lotus and Zelkova carpinifolia in Behshar and other forest areas. On D. lotus the mushroom grew in tufts on crown, stump, and trunk of dead stand or fallen trees, while on Z. carpinifolia it grew on roots, stump, and trunk of living trees. Fruiting bodies were accrued in cluster and counted 10-50 on D. lotus and 3-8 on Z. carpinifolia. The size of fruiting body was smaller on the Z. carpinifolia (Figure 2. D)

Basidiocarps were ochre- yellow, convex at first then flatten measuring $2-5 \mathrm{~cm}$ on $D$. lotus and $4-10 \mathrm{~cm}$ on $Z$. carpinifolia, with thin, elastic flesh and sticky surface during wet weather. Stem was 3-10× 0.3-0.4 cm, narrow downward, yellow above and dark-brown below, velvety, hallow with no ring. Spores were ellipsoid, 5.6-8 × 3.4 micrometers. Under refrigerated condition fruiting bodies were formed after 30 days on media, the caps were very small, $1-1.5 \mathrm{~mm}$, stem $20-30 \mathrm{~mm}$ (Figure 2. C) and in bottle after 25 days, cap 10-30 mm, stem 20-90×1-3mm. (Figure 2. D).

\subsection{Morphology and growth characteristic of mycelium}

Mycelial mat was white at first then turned yellowish brown or brown mixed with white. Pigmentation at the center of colony was distinguishably more than margin. There were more or less visible colored concentric zones, brown to pale from middle to margin. (Figure 2. A) GR and GC were 5.36 and $36.64 \mathrm{~mm} /$ day, respectively. 
Plates were covered with mycelia in 15 days; hyphal width was $2.4-6.4 \mu$. Clamp presents at all septa on sizes $4 \times 1.6-8 \times 4.8 \mu$, in some cases a lateral hypha grows out from a clamp (Figure 3. A).

Chlamydospores were abundant, spherical to ellipsoid on sizes 8-7.2- 11.2-14.4 $\mu$ (Fig.4D). Arthroconidia were in different sizes, cylindrical, rounded, or ellipsoid at the end, liberation (Fig.2A, 4F). There are loops observed in different shapes and sizes. (Figure 3. E)

\subsection{Antagonistic activities}

The interaction between $F$. velutipes and plant pathogenic fungi in dual culture on MEA indicated that $F$. velutipes had different antagonistic effects against plant- pathogenic fungi (Table 2). The mushroom had strong antagonistic effect on Ophiostoma ulmi. In this case complete replacement of plant-pathogenic fungi by $F$. velutipes was recorded after initial deadlock (Fig. 5D); however it had the less effect on Bipolaris sorokiniana (Fig.5A). In this case deadlock in distance was recorded. For three other partial replacement after initial deadlock were happened (Fig. 5B, C, E, F).

\section{Discussion}

According to available literature, F. velutipes hasn't been reported on D. lotus (Ebenaceae) and Zelkova carpinifolia (Ulmaceae) in Iran, and hereby reporting as new hosts for these spices.

Comparing growth characteristics of our strain with 23 strains from different parts of the word Badalyan et al., 2006] it is close to strains SBR-9 from ash tree in Russia. Comparing antagonistic effect of our strain with the strain of III $2 F v$ [Badalyan et al., 2002] on cereal disease agents, it performed similarly on $F$. culmurum and differed on $B$. sorokiniana. This study showed $F$. velutipes had antifungal and antagonistic effect on agents of this disease under in vitro conditions. This open up the possibility of using this mushroom for the management of the diseases in future. The existence of this mushroom during winter, in which no other edible species found, is another credible characteristic to attract professionals and amateurs edible mushroom explorers towards it.

Usage percapita of edible mushroom in Iran is too low, about $200 \mathrm{~g}$, whereas this is $1200-1500 \mathrm{~g}$ for world, it can be because of low variation in species of edible mushrooms production (2-3 spices). Production and introducing of these species can attract new consumer to edible mushrooms. In most villages of north of Iran, sawdust can be used for production of this mushroom, a way for creating more jobs, therefore more welfare

\section{References}

Badalyan S.M. (2004). Screening of antifungal activity of several Basidiomycetes mushrooms. Probl Med Mycol, 6, 18-26.

Badalyan S.M., Hughes H.W. \& Sakeyan C.Z. (2006). Morphology, growth characteristics, and genetic variation of the edible medicinal mushroom Flammulina velutipes collections. Int J Med Mushr., 8, 263-278.

Badalyan S.M., Innocenti G. \& Garibyan N.G. (2002). Antagonistic activity of xylographic mushroom against pathogenic fungi of cereal in dual culture. Phytopathol Mediterr., 41, 220-225.

Badalyan S.M., Sakeyan C.Z. (2004). Morphological and growth characteristic of mycelium of several medicinal mushroom (Aphyllophoromycetideae). Int J Med Mushr., 6, 347-360.

Badalyan, S.M. \& Hambardzumyan L.A. (2001). Investigation of immune -modulation activity of medicinal mushroom Flammulina velutipes in vitro cytokines induction by fruiting body extract. Int J Med Mushr., 3, 110 .

Buchalo A.S. (1988). The higher edible Basidiomycetes in pure culture. Nauk. Dumaka press, Kiev, 143 pp.

Chang S.T. \& Miles P.G. (1987). Historical record of the early cultivation of Lentinus in China. Mushr Jf Tropic, 7, 31-37.

Chang S.T. \& Miles P.G. (2004). Mushrooms, cultivation, nutritional value, medicinal effect and environmental effect. Sec. Edit. P. 451.

Hughes K.W., McGhee L.L., Methven A.S., Johnson J.E. \& Peteresen R. H. (1999). Patterns of geographic speciation in the genus Flammulina based on sequences of ribosomal ITSI-5.8S-ITS2area. Mycol. 91, 978-986.

Ikekawa T. (2001). Beneficial affects of edible and medicinal mushrooms on health care. Int J Med Mushr., 3, 291-298.

Komatsu N., Terakawa H., Nakanishi K. \& Watanabe Y. (1963). Flammuline a basic protein of Flammulina velutipes with an antitumor activity. Journal of Antibiotics., Ser. A. 16, 139-143.

Leung M.Y.K., Fung K.P. \& Choy Y.M. (1997). The isolation and characterization of an immunomodulatory and antitumor Polysaccharide preparation from Flammulina velutipes. Immunopharmacol., 35, $255-263$. 
Melo, M.R., Paccola-Meirelles, L.D., Ishikawa, N.K. \& Faria, T.J.D. (2009). Influence of Flammulina velutipes mycelia culture conditions on antimicrobial metabolite production Mycosci., 50, 78-81.

Nobles, M. (1965). Identification of cultures of wood inhabiting Hymenomycetes. Can J Bot., 43, 1097-1139.

Redhead S.A. \& Peteresen R.H. (1999). New species, varieties, and combinations in the genus Flammulina. Mycologia., 71, 285-294.

Saber M. (1998). Collection and identification collybioid mushrooms in Iran, $13^{\text {th }}$ Iranian plant protection congress P.278.

Sageb-talebi K., Sajedi T. \& Yazdian F. (2005). Forest of Iran. Research Institute of Forests and Research and Rangelands. $28 \mathrm{p}$.

Smith, J.E. \& Sullivan, R. (2004). The western approach to medicinal mushrooms. K M T I L. Sci J. 4 (1) (www.ac.Th/science/Journal/Jan2004/Mushrooms.pdf).

Stalpers J.A. (1978). Identification of wood -inhibition Aphyllophorales in pour cultures. Stud Mycol. 16, 248 P.

Wasser, S.P. \& Weise, A.L. (1999). Medicinal properties of substance occurring in higher Basidiomycetes mushrooms: Current perspective (review). Int J Med Mushr., 1, 31-62.

Zokaie M. (2001). Study on Agaricales mushrooms in Mashhad. Rost, Botal J Iran., 2, 7-13.

Table 1. List of Plant pathogenic fungi and their sources used in this study for antifungal activity of $F$. velutipes against them

\begin{tabular}{|c|l|l|}
\hline & Plant pathogenic fungi & Source \\
\hline 1 & Fusarium oxysporum & isolated from Albizia julibrissin in Challus forest area \\
\hline 2 & Ophiostoma ulmi & isolated from Ulmus glabra In Sangedeh forest area \\
\hline 3 & Pestalotiopsis funerea & isolated from Sequoia sempervirens in Behshar area \\
\hline 4 & Fusarium culmurum LM 2091 & $\begin{array}{l}\text { received from Laboratory of Fungi biology and } \\
\text { biotechnology, Yerevan State University }\end{array}$ \\
\hline 5 & Bipolaris sorokiniana strain Bs-I & $\begin{array}{l}\text { received from Laboratory of Fungi biology and } \\
\text { biotechnology, Yerevan State University }\end{array}$ \\
\hline
\end{tabular}

Table 2. Antagonistic scare and competitive reaction between mycelium of $F$. velutipes and plan- pathogenic fungi in paring on MEA.

\begin{tabular}{|l|l|c|c|}
\hline Plant -pathogenic fungi & Agent of disease & Type of reaction & Antagonistic Score \\
\hline Fusarium oxysporum & Wilt diseases of silk tree & $\mathrm{C}_{\mathrm{A} 1}$ & 3.5 \\
\hline Ophiostoma ulmi & Dutch elm disease of elm trees & $\mathrm{C}_{\mathrm{A} 2}$ & 4.5 \\
\hline Pestalotiopsis funerea & Die back of conifers & $\mathrm{C}_{\mathrm{A} 1}$ & 3.5 \\
\hline Fusarium culmurum & Foot root of cereal & $\mathrm{C}_{\mathrm{A} 1}$ & 3.5 \\
\hline Bipolaris sorokiniana & Foot root of cereal & $\mathrm{B}$ & 2 \\
\hline
\end{tabular}

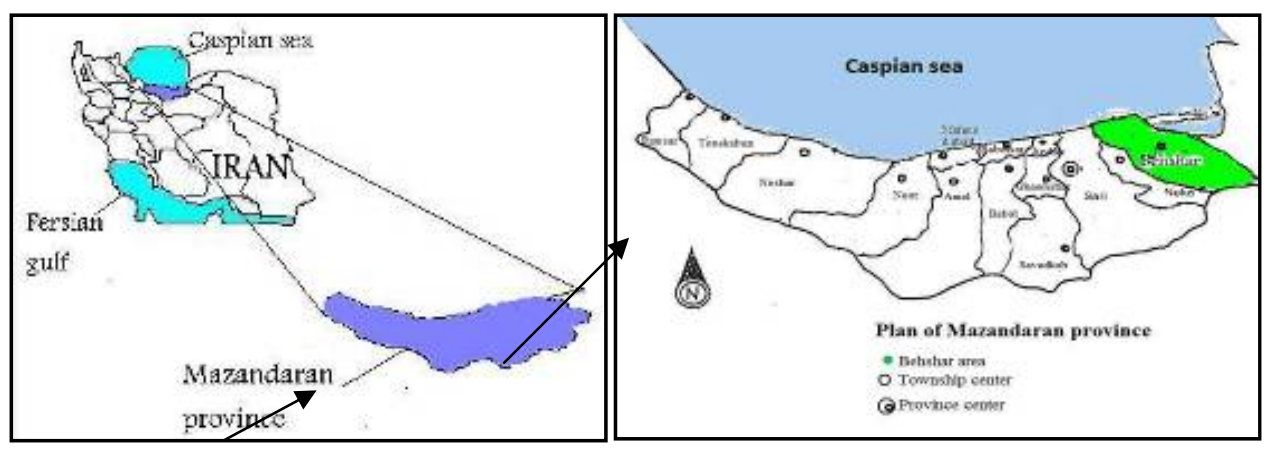

Figure 1. Iran and location of Mazandaran province (left), plan of Mazandaran and location of Behshar area (right) 

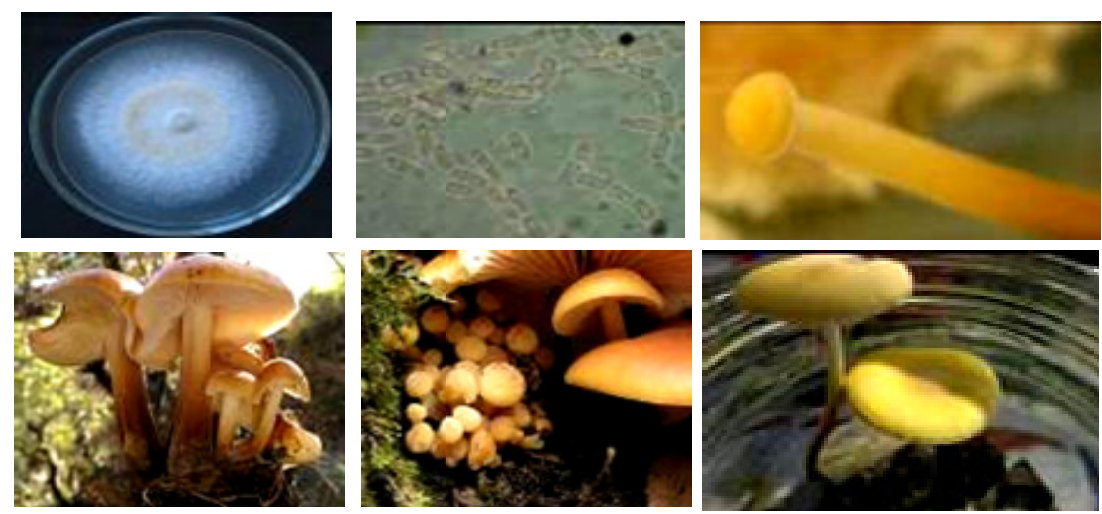

Figure 2. F. velutipes: colony (A), Arthroconidia (B), Fruiting body; on tube culture (C), on Z. carpinifolia (D), on D. lotus (E) on pieces of host's wood in bottle.

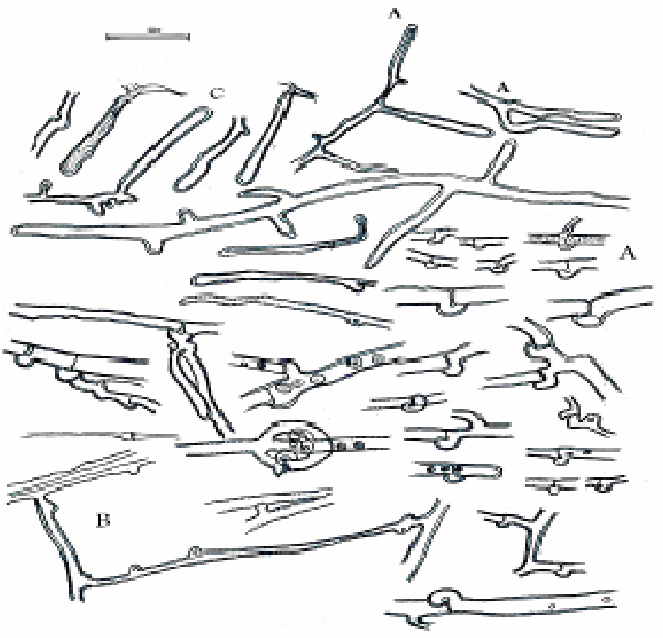

Figure 3. Hyphae and clamp (A), Anastomos1s(B), seta and setal hyphae $(\mathrm{C})$
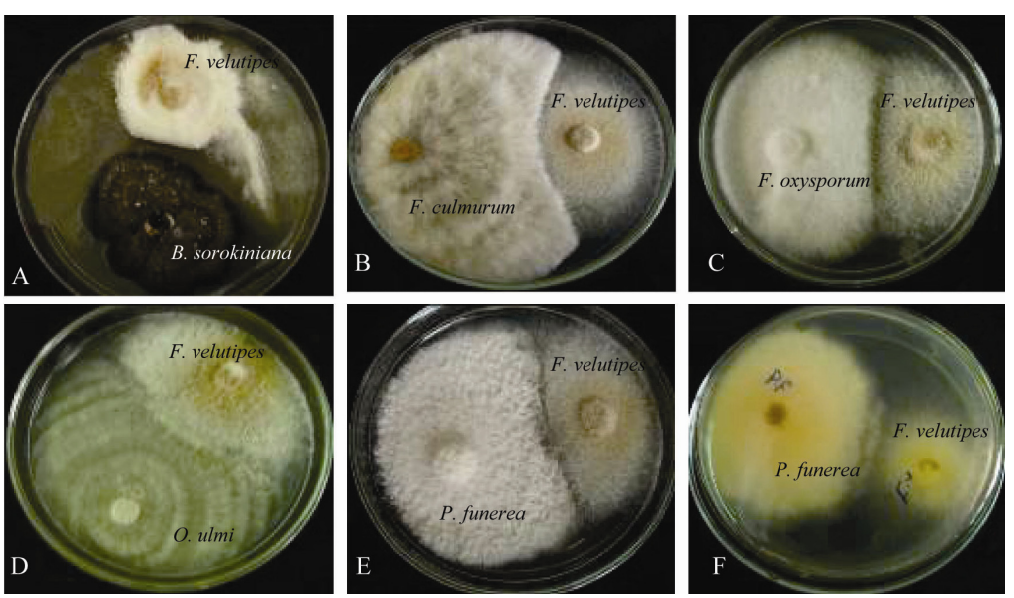

Figure 5. Antagonistic effect of $F$. velutipes on plant-pathogenic fungi in dual culture. (A) Deadlock at distance on B. sorokiniana .(B,C, E, F) Partial replacement after initial deadline on F. culmurum, F. oxysporum, $P$. funerea respectively. (D) complete replacement after initial Deadline. 